\title{
Integrated versus segregated accounting and the magnitude effect in temporal discounting
}

\author{
RANDOLPH C. GRACE and ANTHONY P. MCLEAN \\ University of Canterbury, Christchurch, New Zealand
}

\begin{abstract}
Temporal discounting rates in humans generally decrease as the amount of reward increases, a phenomenon known as the magnitude effect. In the present study, we examined whether temporal discounting and the magnitude effect are related to segregation of choices in terms of gains or losses for waiting for or expediting receipt of a reward. Subjects $(N=24)$ responded to a series of hypothetical choices about amounts of money available either immediately or after a delay. The immediate and delayed amounts either were presented as integrated amounts in the baseline condition or were segregated as differential gains or losses for choosing delayed or expedited consumption (delay and speedup conditions, respectively). Temporal discounting rates decreased in the segregated conditions, in accord with the standard discounted utility model but contrary to the hypothesis that the subjects were choosing on the basis of reward differentials in the baseline condition. The size of the magnitude effect was comparable in the baseline and the delay conditions but decreased in the speed-up condition. These results challenge explanations of the magnitude effect in terms of an increasing proportional sensitivity property of the utility function (Loewenstein \& Prelec, 1992) and the hypothesis that subjects choose on the basis of differentials even when the rewards are presented as integrated amounts.
\end{abstract}

We often make choices in which one or more potential outcomes may be delayed. If an employee receives a Christmas bonus check for $\$ 2,000$, should he or she deposit the money in a pension plan or spend it on a holiday? The long-run benefit of investing the money in the pension plan is greater, yet the employee may still book travel reservations, because consumption occurs in the near term. Research on intertemporal choice attempts to identify the factors that determine behavior allocation in such cases; the process whereby the value of an outcome decreases as a function of its delay is called temporal discounting (Chapman, 1998). According to the normative model for temporal discounting, the discounted utility (DU) model, the value $(V)$ of a reward $x$ received after delay $t$ is given by

$$
V=v(x) \delta(t),
$$

where $v$ is a utility function and $\delta$ is a discounting function that was originally assumed to be exponential (note that $\delta(0)=1$, and $\delta(t) \rightarrow 0$ as $t \rightarrow^{\circ}$ ). However, empirical evidence indicates that a hyperbolic function provides a better account of the data (Ainslie, 1974; Kirby, 1997; Mazur, 1984; Myerson \& Green, 1995).

One of the most pervasive phenomena in intertemporal choice is the so-called magnitude effect: The observed

We thank Rebekah Gibson and Matthew Healey for help with data collection. Address correspondence to R. C. Grace, Department of Psychology, University of Canterbury, Private Bag 4800, Christchurch, New Zealand (e-mail: randolph.grace@ canterbury.ac.nz). rate of temporal discounting decreases as the reward amount increases. To illustrate, someone may be indifferent between receiving gifts of $\$ 400$ now or $\$ 500$ in 1 year, yet strongly prefer $\$ 15,000$ in 1 year to $\$ 12,000$ now. Although in each case the actual return for waiting is $25 \%$, the preferences differ depending on the absolute reward sizes involved, which implies that the discounting rate for $\$ 15,000$ is less than that that for $\$ 500$. The magnitude effect is a robust finding, having been obtained with humans making hypothetical choices about money (Benzion, Rapoport, \& Yagil, 1989; Green, Myerson, \& McFadden, 1997; Myerson \& Green, 1995; Thaler, 1981), health outcomes (Chapman, 1996), and tipping scenarios (Chapman \& Winquist, 1998). However, despite the many similarities observed across species in research on choice between delayed rewards (e.g., Rodriguez \& Logue, 1988), the magnitude effect may be restricted to humans. Grace (1999) found that temporal discounting rates did not depend on reward amount in an experiment with pigeons choosing between different delays to food (see also Green, Myerson, Holt, Slevin, \& Estle, 2004; Ong \& White, 2004).

Various explanations have been offered for the magnitude effect. Thaler (1981) proposed that large and small amounts are placed in separate "mental accounts," with small amounts targeted for immediate consumption and large amounts reserved for savings. However, Green et al. (1997) found that the discounting rate decreased continuously as a function of reward amount, which seems implausible in terms of mental accounts. An alternative explanation for the magnitude effect is that it reflects characteristics of the utility function for money-namely, 
an increasing proportional sensitivity property (IPS; Loewenstein \& Prelec, 1992):

$$
\frac{v(x)}{v(q)}<\frac{v(\alpha x)}{v(\alpha q)}, \text { for } x>q>0, \alpha>1
$$

Equation 2 states that the ratio of values (or utilities) for two amounts of money in constant ratio increases as the absolute amounts increase. In the example above, the ratio of the values of $\$ 15,000$ and $\$ 12,000$ is greater than for $\$ 500$ and $\$ 400$; hence, the implied discounting rate is less for the larger amounts. Chapman and Winquist (1998) argued that evidence for the magnitude effect in nontemporal choice scenarios, such as restaurant tips, supported the hypothesis that the magnitude effect was due to intrinsic properties of the utility function - namely, IPS. Thus, IPS can reconcile the magnitude effect with the standard DU model for intertemporal choice (Loewenstein \& Prelec, 1992).

But does the DU model correspond to how subjects make choices in discounting experiments? In many studies, subjects are offered a series of hypothetical choices, and psychophysical techniques are used to determine indifference points - the amount of money available immediately that is judged as being equivalent in value to a delayed reward. For example, a subject might be asked to choose between $\$ 400$ now or $\$ 500$ in 1 year. According to the DU model, the response would be determined by the values of the alternatives: $v(\$ 400) \delta(0)$ and $v(\$ 500)$ $\delta$ (1 year). Yet the subject is are free to interpret the question in various ways. For example, he or she might view the choice as whether or not to earn an extra $\$ 100$ by waiting 1 year. Conversely, he or she might regard the question in terms of how much money must be foregone $(\$ 100)$ to receive some amount immediately. In both cases, the subject would effectively be shifting the reference points and then deciding whether the reward differential justified delaying or expediting consumption.

Note that the differential will increase as the absolute reward amounts increase, with their ratio held constant. For example, the differential is $\$ 3,000$ for the choice above between $\$ 12,000$ now or $\$ 15,000$ in 1 year, as compared with $\$ 100$ for a choice between $\$ 400$ now and $\$ 500$ in 1 year. The magnitude effect would result if the subject was more likely to choose on the basis of reward differentials, rather than discounted value in accord with the DU model, when the differential is large. This may be called the reward differential hypothesis. Previous studies have not varied how the immediate and delayed amounts are presented, and so it is unknown whether this hypothesis might account for the magnitude effect.

The importance of reference points for intertemporal choice was first noted by Loewenstein (1988). He found that discounting rates increased when subjects, who were anticipating immediate or delayed consumption of a durable good (a VCR with a list price of \$300), were asked to name fair premiums for delaying or expediting receipt of the VCR, respectively. The results were consistent with a reference point model in which how a question is framed can affect discounting rates, provided that the subjects adapt to a new consumption level. According to his model, the subjects required a large premium to delay consumption, because they had adapted to immediate receipt of the VCR; delaying receipt for 1 year was experienced as an immediate loss, compensated by a delayed gain.

In our experiment, we manipulated how choices were presented in a standard temporal discounting paradigm. In the baseline condition, indifference points for rewards of $\$ 500$ and $\$ 15,000$ were obtained for a range of delays. This condition was similar to those in previous studies, in that the monetary options were presented directly as integrated amounts. The subjects also completed two additional conditions, in which all questions were explicitly posed in terms of reward differentials. Standard amounts for these conditions were $\$ 400 / \$ 12,000$ and $\$ 600 /$ $\$ 18,000$. In the delay condition, the immediate amount was displayed as in the baseline condition, but the delayed amount was presented as a differential reward for delaying consumption. In the speed-up condition, the delayed amount was similar to that in the baseline condition, but the immediate amount was presented as a differential cost for expediting consumption.

The standard DU model predicts that temporal discounting rates should be lower in the delay and speed-up conditions, as compared with baseline, because of decreasing marginal utility (see Appendix A). In addition, if the utility function is steeper for losses than for gains (Kahneman \& Tversky, 1979), the discounting rates should be lowest overall in the speed-up condition. The DU model with an IPS utility function predicts that the size of the magnitude effect-defined as the ratio between the immediate-amount indifference points for the large and the small delayed rewards-should be greater in the delay and speed-up conditions, as compared with the baseline condition (see Appendix B). Moreover, the DU model with IPS predicts that the difference between the size of the magnitude effects in the delay or speed-up condition, relative to the baseline condition, should be correlated with the reduction in discounting rates in those conditions. By contrast, if subjects are choosing on the basis of reward differentials in the baseline condition, there should be no difference in overall discounting rates between the baseline and the delay and speed-up conditions. The reward differential hypothesis also predicts that the size of the magnitude effect should be the same in all the conditions.

\section{METHOD}

\section{Subjects}

Twenty-four students were recruited from courses in the Department of Psychology, University of Canterbury. There were $10 \mathrm{fe}-$ males and 14 males, and the average age was 24.9 years. All the subjects received a $\$ 5$ lottery ticket in return for their participation.

\section{Procedure}

The subjects were tested in a quiet room, seated individually in front of a computer. Instructions displayed on the screen explained 


\section{Baseline}
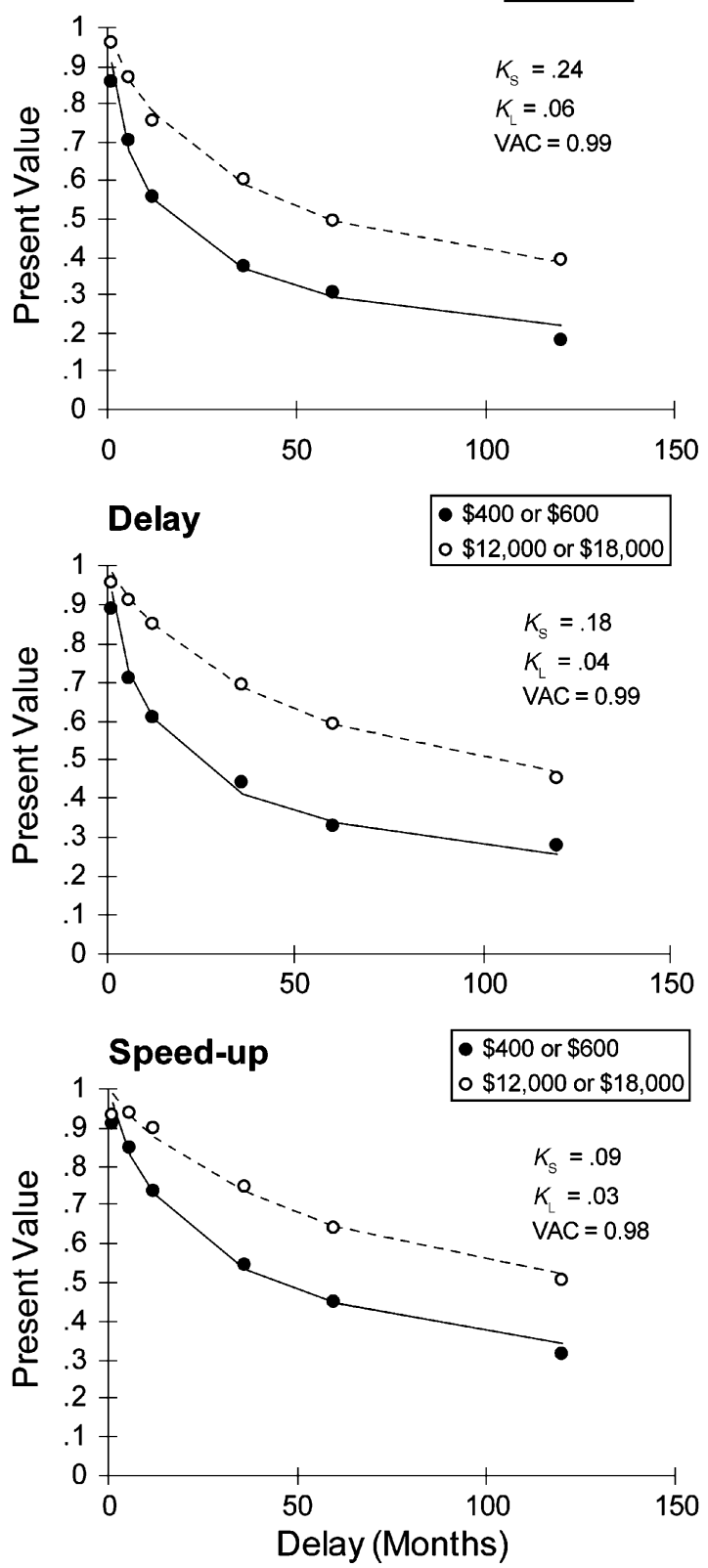

Figure 1. Normalized indifference points (present value) as a function of delay for all three conditions (baseline, delay, and speed-up), averaged across subjects. Data for the small-amount standard rewards are shown by filled circles; data for the largeamount standard rewards are shown by unfilled circles. The dashed and solid lines indicate the fit of the generalized hyperbola (Equation 3) to the data. The best-fitting discount rate parameters for the small- $\left(K_{S}\right)$ and large- $\left(K_{L}\right)$ amount standard data and the variance accounted for (VAC) are listed under the legend in each panel.

Because the estimated $K$ values were positively skewed, a logarithmic transformation was performed prior to statistical analysis. In the following, we used the estimated
$K$ values from the fits of Equation 3 with $s$ allowed to vary. All analyses were replicated with $K$ values from fits with $s=1$, and the results were highly similar.

To check the validity of the counterbalancing procedure, we conducted repeated measures ANOVAs for the delay and speed-up conditions, depending on whether the amounts were $\$ 400 / \$ 12,000$ or $\$ 600 / \$ 18,000$. There were no significant main effects or interactions involving the counterbalanced amounts. Thus, the results were pooled across $\$ 400 / \$ 12,000$ and $\$ 600 / \$ 18,000$ for the delay and speed-up conditions for subsequent analyses.

$\log K$ discounting rates were entered into a repeated measures ANOVA, with condition and amount as factors. The average $\log K$ values for each condition and reward amount (large/small) are shown in Figure 2. The main effect of condition was significant $[F(2,46)=8.28, p<$ .001]. Planned comparisons showed that the discounting rates in the baseline condition were greater than the averages for the delay and speed-up conditions $[F(1,23)=$ $15.52, p<.001]$ and were greater in the delay than in the speed-up condition $[F(1,23)=5.10, p<.05]$. The ordering of discounting rates across conditions (baseline $>$ delay $>$ speed-up) is consistent with the standard DU model for intertemporal choice but is contrary to the prediction of the reward differential hypothesis. The main effect of magnitude was also significant $[F(1,23)=$ $70.06, p<.001]$, confirming the magnitude effect: Discounting rates were reduced with larger amounts. The condition $\times$ magnitude interaction did not reach significance $[F(2,46)=0.86, n . s]$. The lack of interaction is evident in Figure 1, where the difference between the fits of Equation 3 to the small- and large-magnitude amounts (dashed and solid lines, respectively) appears approximately constant across the three conditions.

We derived a measure of the size of the magnitude effect consistent with the analysis in Appendix B. First, the $\mathrm{PV}$ ratio (large/small) was calculated for each delay within a particular condition. The ratios were transformed logarithmically to render equal ratios as equal intervals. Finally, the log PV ratios were averaged across delays $(N=6)$ to give a magnitude effect size measure (ME) for each condition:

$$
\mathrm{ME}=\frac{\left(\frac{\sum_{i=1}^{N} \log \mathrm{PV}_{\text {large } i}}{\mathrm{PV}_{\text {small } i}}\right)}{N} .
$$

Average magnitude effect sizes are shown in Figure 3. A one-way repeated measures ANOVA yielded a significant effect of condition $[F(2,46)=3.72, p<.05]$. Planned comparisons showed that the magnitude effect was reduced in the speed-up condition, as compared with the baseline and delay conditions $[F(1,23)=5.61, p<.05$, and $F(1,23)=6.34, p<.05$, respectively]. However, the magnitude effect size did not differ between the baseline and the delay conditions. These results are contrary to the 


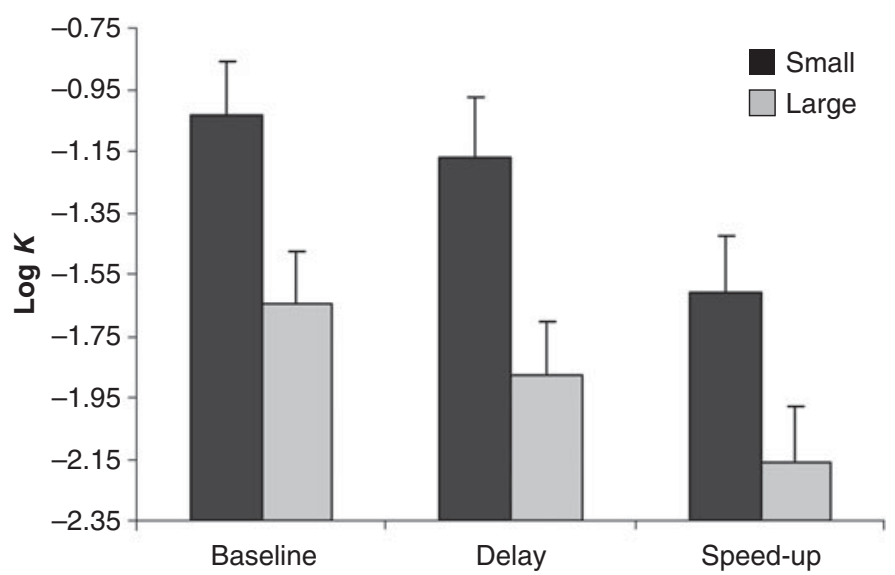

Figure 2. Average discounting rates $(\log K)$ for all conditions and both reward amounts (small and large). Bars indicate one standard error.

prediction of the DU model with IPS, which predicted that the size of the magnitude effect should have increased in the delay and speed-up conditions, relative to baseline.

A second prediction of the standard DU model with IPS as an explanation for the magnitude effect is that the size of the magnitude effect in the delay and speed-up conditions should be positively related to the reduction in discounting rates in those conditions (see Appendix B). More specifically, there should be a positive correlation, within subjects, between the difference between average $\log K$ values for the baseline condition and the delay or speed-up condition and the size of the magnitude effect (as computed by Equation 4) within the delay and speed-up conditions. For the delay condition, the correlation was negative but not significant $(r=-.21$, n.s.). The correlation for the speed-up condition was also not significant $(r=-.08$, n.s. $)$.

\section{DISCUSSION}

Our experiment compared temporal discounting rates for subjects choosing between hypothetical amounts of money available immediately or after a delay. Large and small amounts were studied over the same range of delays so that we could investigate the magnitude effectthe finding that discount rates vary inversely with reward amount. In previous research using psychophysical procedures to estimate indifference points, the delayed and the immediate amounts have been presented as integrated totals. This was the procedure used in our baseline condition. However, we were interested in whether segregated accounting - specifically, presenting the results in terms of differential gains or losses for delaying or expediting consumption, respectively-would affect discounting rates and the magnitude effect. One potential explanation for the magnitude effect is the reward differential hypothesis - that subjects may choose on the basis of differential gains or losses even if the amounts are pre- sented as integrated totals and that the likelihood of their responding in this way increases as the differentials increase. According to this view, discounting rates should have been similar, regardless of whether integrated or segregated rewards were presented. However, we found that discounting rates were reduced in the delay and speed-up conditions, as compared with the baseline condition (see Figure 2). Given that discounting rates were greater for small than for large rewards across all the conditions, the reward differential hypothesis seems untenable as an explanation for the magnitude effect.

The reduction in discounting rates in the delay and speed-up conditions was consistent with the predictions of the standard DU model for intertemporal choice (Equation 1). According to the DU model, segregating rewards should effectively make them more valuable, because of decreasing marginal utility, leading to a reduction in discounting rate (see Appendix A). With the additional assumption of a steeper utility function for losses than for gains, the DU model predicts that discounting rates should be lower in the speed-up than in the delay condition. This

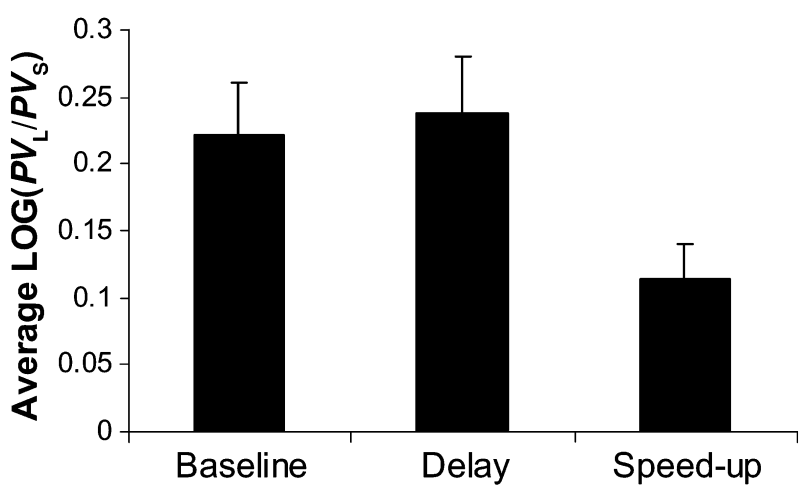

Figure 3. Average magnitude effect sizes for all conditions, defined according to Equation 4. Bars indicate one standard error. 
prediction was also confirmed by our data. However, the standard DU model is unable to account for the magnitude effect.

One way to reconcile the magnitude effect with the DU model is the assumption that the utility function is characterized by an IPS property (Equation 2; Loewenstein \& Prelec, 1992). The DU model with IPS makes two predictions for our experiment: that the size of the magnitude effect should have been greater in the delay and speed-up conditions and that there should have been a positive correlation between the reduction in discount rates in the delay and speed-up conditions, as compared with the baseline condition, and the size of the magnitude effect (see Appendix B). Neither prediction was confirmed. The size of the magnitude effect was smallest in the speed-up condition and was comparable in the baseline and delay conditions (see Figure 3 ). The correlations between reduction in discounting rates and magnitude effect size were not significantly different from zero. Thus, the present data pose a challenge for IPS as an explanation of the magnitude effect within the standard DU model.

Because our subjects made different judgments when rewards were presented as unitary amounts, as opposed to segregated into a base amount plus a gain (for waiting) or a cost (for immediate consumption), it appears that people do not routinely reframe self-control choices in this way. Our results indicate that it would often be beneficial for them to do so. People desiring to exert greater self-control in everyday life choices often discount delayed rewards so rapidly that a small but immediate outcome is preferred over a larger, delayed one. Calculating the absolute gain with delayed consumption (or the absolute cost of immediate consumption) is easily possible when investment or purchasing decisions are made, for example, and our results indicate that doing so might increase self-control by decreasing the discounting rate for delayed outcomes. Although we are unaware of this tactic's having been exploited to enhance self-control in applied settings, there are many examples in which reward segregation is used in marketing to influence choice. Rather than reduce the cost, marketers often add a bonus item to an advertised product, presumably increasing choices of expenditure over saving. The effectiveness of such manipulations may be related to the often-reported difference between sensitivity to variations in relative rate of reinforcement and that to variations in relative magnitude of reinforcement in studies of nonhuman choice (e.g., McLean \& Blampied, 2001).

Thus, our results show that presenting choice options in terms of segregated gain or loss differentials can affect discounting rates and the size of the magnitude effect in intertemporal choice scenarios. The results were contrary to the predictions of two potential explanations for the magnitude effect: the reward differential hypothesis and IPS. The standard DU model correctly predicted the decreases in discounting rates for the delay and speed-up conditions, and it may be that an appropriate extension of this model will ultimately provide an adequate account of the magnitude effect for intertemporal choice.

\section{REFERENCES}

Ainslie, G. W. (1974). Impulse control in pigeons. Journal of the Experimental Analysis of Behavior, 21, 485-489.

BENZION, U., RAPOPORT, A., \& YAGIL, J. (1989). Discount rates inferred from decisions: An experimental study. Management Science, 35, 270-284.

Chapman, G. B. (1996). Temporal discounting and utility for health and money. Journal of Experimental Psychology: Learning, Memory, \& Cognition, 22, 771-791.

Chapman, G. B. (1998). Sooner or later: The psychology of intertemporal choice. In D. Medin (Ed.), The psychology of learning and motivation (Vol. 38, pp. 83-113). San Diego: Academic Press.

Chapman, G. B., \& Winguist, J. R. (1998). The magnitude effect: Temporal discount rates and restaurant tips. Psychonomic Bulletin \& Review, 5, 119-123.

GRACE, R. C. (1999). The matching law and amount-dependent exponential discounting as accounts of self-control choice. Journal of the Experimental Analysis of Behavior, 71, 27-44.

GreEn, L., Fristoe, N., \& Myerson, J. (1994). Temporal discounting and preference reversals in choice between delayed outcomes. Psychonomic Bulletin \& Review, 1, 383-389.

Green, L., Myerson, J., Holt, D. D., Stevin, J. R., \& Estle, S. J. (2004). Discounting of delayed food rewards in pigeons and rats: Is there a magnitude effect? Journal of the Experimental Analysis of Behavior, 81, 39-50.

GreEn, L., Myerson, J., \& McFadden, E. (1997). Rate of temporal discounting decreases with amount of reward. Memory \& Cognition, 25, 715-723.

KAHNEMAN, D., \& TVERsky, A. (1979). Prospect theory: An analysis of decision under risk. Econometrica, 47, 263-291.

KIRBY, K. N. (1997). Bidding on the future: Evidence against normative discounting of delayed rewards. Journal of Experimental Psychology: General, 126, 54-70.

LoEWENSTEIN, G. (1988). Frames of mind in intertemporal choice. Management Science, 34, 200-214.

Loewenstein, G., \& Prelec, D. (1992). Anomalies in intertemporal choice: Evidence and an interpretation. In G. Loewenstein \& J. Elster (Eds.), Choice over time (pp. 119-145). New York: Russell Sage Foundation.

Mazur, J. E. (1984). Tests for an equivalence rule for fixed and variable reinforcer delays. Journal of Experimental Psychology: Animal Behavior Processes, 10, 426-436.

MCLEAN, A. P., \& BLAMPIED, N. M. (2001). Sensitivity to relative reinforcer rate in concurrent schedules: Independence from relative and absolute reinforcer duration. Journal of the Experimental Analysis of Behavior, 75, 25-42.

MYERSON, J., \& GREEN, L. (1995). Discounting of delayed rewards: Models of individual choice. Journal of the Experimental Analysis of Behavior, 64, 263-276.

ONG, E. L., \& White, K. G. (2004). Amount-dependent temporal discounting? Behavioural Processes, 66, 201-212.

Rodriguez, M. L., \& Logue, A. W. (1988). Adjusting delay to reinforcement: Comparing choice in pigeons and humans. Journal of Experimental Psychology: Animal Behavior Processes, 14, 105-117.

THALER, R. (1981). Some empirical evidence on dynamic inconsistency. Economics Letters, 8, 201-207. 


\section{APPENDIX A}

In the following, we present a proof that the DU model predicts that discounting rates should be reduced when choices are framed as gains/losses for delaying/expediting consumption (as compared with the baseline condition, in which immediate and delayed options are presented as unitary amounts). ${ }^{\mathrm{A} 1}$

For each standard amount and delay, an indifference point (i.e., the immediate amount equal in value to the delayed amount) is determined. Thus, for the three conditions, the following equalities must hold:

$$
\begin{array}{ll}
v\left(x_{\mathrm{i}}\right)=v\left(x_{\mathrm{d}}\right) \delta(t) & \text { [baseline], } \\
v\left(x_{\mathrm{iD}}\right)=\left[v\left(x_{\mathrm{iD}}\right)+v\left(x_{\mathrm{d}}-x_{\mathrm{iD}}\right)\right] \delta(t) & \text { [delay], } \\
v\left(x_{\mathrm{d}}\right)+v\left[\left(x_{\mathrm{iS}}-x_{\mathrm{d}}\right)\right]=v\left(x_{\mathrm{d}}\right) \delta(t) & \text { [speed-up], }
\end{array}
$$

where $x_{\mathrm{i}}, x_{\mathrm{iD}}$, and $x_{\mathrm{iS}}$ are the indifference points from the baseline, delay, and speed-up conditions, respectively, and $x_{\mathrm{d}}$ is the delayed amount. Consider the delay condition first. By decreasing marginal utility, $v\left(x_{\mathrm{iD}}\right)+$ $v\left(x_{\mathrm{d}}-x_{\mathrm{iD}}\right)>v\left(x_{\mathrm{d}}\right)$. Therefore, $v\left(x_{\mathrm{iD}}\right)>v\left(x_{\mathrm{i}}\right)$, so the discounting rate implied by the indifference point $\left(x_{\mathrm{i}}\right)$ is lower in the delay condition.

For the speed-up condition, the left-hand side of Equation A3 must equal the left-hand side of the baseline equality, $v\left(\mathrm{x}_{\mathrm{i}}\right)$. As in the delay condition, decreasing marginal utility implies that $x_{\mathrm{iS}}>x_{\mathrm{i}}$, so the discounting rates in the speed-up condition are reduced, as compared with baseline. If we further assume that the utility function is steeper for losses than for gains-that is, $-v(-x)>v(x)$-the discounting rates will be also be lower than those in the delay condition.

\section{NOTE}

A1. Loewenstein (1988) showed that according to the standard DU model, the difference between the price that someone was willing to pay for a durable good (e.g., a VCR) received immediately and the price for the same good received after 1 year should be equal to the amount required to delay or expedite consumption. The implication is that the standard DU model predicts that the discounting rate should be the same in all three conditions of the present experiment, contrary to Appendix A. However, in applying the DU model, Loewenstein assumed that utilities were based on integrated amounts and did not apply the utility function separately to the immediate/delayed amount and the differential. Because those amounts are displayed separately in the delay and the speed-up conditions in the present experiment, we have assumed that subjects will value them individually and have derived predictions of the DU model accordingly.

\section{APPENDIX B}

In the following, we present a proof that the standard DU model with IPS predicts that the size of the magnitude effect should be greater in the delay and speed-up conditions than in the baseline condition. Moreover, the size of the magnitude effect in the delay and speed-up conditions should be positively correlated with the reduction in the discounting rate in those conditions, as compared with baseline.

Recall that the IPS property requires that the ratio of the values of two amounts increases when the amounts are multiplied by a positive constant, $\alpha$ :

$$
\frac{v(\alpha x)}{v(\alpha q)}>\frac{v(x)}{v(q)} .
$$

Consider the baseline condition first. Indifference points are determined for $x_{\mathrm{d}}$ and $\alpha x_{\mathrm{d}}$ (i.e., small and large rewards). For $x_{\mathrm{d}}$,

$$
v\left(x_{\mathrm{i}}\right)=v\left(x_{\mathrm{d}}\right) \delta(t) .
$$

For the large reward, the indifference point for $\alpha x_{\mathrm{d}}$ will be greater than $\alpha x_{\mathrm{i}}$ by the IPS property, depending on $\alpha$ and $x_{\mathrm{i}}$ for a given utility function. In general,

$$
v\left[\alpha x_{\mathrm{i}}+m\left(\alpha, x_{\mathrm{i}}\right)\right]=v\left(\alpha x_{\mathrm{d}}\right) \delta(t),
$$

where $m$ is a positive, monotonically increasing function of both $\alpha$ and $x_{\mathrm{i}}$. The size of the magnitude effect for the baseline condition $\left(\mathrm{ME}_{\mathrm{Base}}\right)$ is then given by the ratio of indifference points for the large and the small rewards:

$$
\mathrm{ME}_{\text {Base }}=\frac{\alpha x_{\mathrm{i}}+m\left(\alpha, x_{\mathrm{i}}\right)}{x_{\mathrm{i}}}=\alpha+m\left(\alpha, x_{\mathrm{i}}\right) \text {. }
$$




\section{APPENDIX B (Continued)}

For the delay condition, let $x_{\mathrm{iD}}$ be the indifference point for the small reward. Then, by logic similar to that above, the size of the magnitude effect for the delay condition is given by

$$
\mathrm{ME}_{\text {Delay }}=\frac{\alpha x_{\mathrm{iD}}+m\left(\alpha, x_{\mathrm{iD}}\right)}{x_{\mathrm{iD}}}=\alpha+m\left(\alpha, x_{\mathrm{iD}}\right) .
$$

But $x_{\mathrm{iD}}>x_{\mathrm{i}}$ by decreasing marginal utility (see Appendix A), so $\mathrm{ME}_{\text {Delay }}>\mathrm{ME}_{\mathrm{Base}}$. Moreover, the size of the difference between $\mathrm{ME}_{\text {Delay }}>\mathrm{ME}_{\mathrm{Base}}$ depends on the difference between $x_{\mathrm{iD}}$ and $x_{\mathrm{i}}$-that is, the reduction in discounting rate between the delay and the baseline conditions. A similar argument holds for the speed-up condition, because $x_{\mathrm{iS}}>x_{\mathrm{i}}$. Thus, the DU model with IPS predicts that the size of the magnitude effect should be greater in the delay and speed-up conditions than in the baseline condition, and that the difference in the effect sizes should be correlated with the reduction in discounting rate.

(Manuscript received April 28, 2004;

revision accepted for publication October 5, 2004.) 